\title{
ESPECIALIZACIÓN CULTURAL Y ESTRUCTURA SOCIAL DE LAS COMUNIDADES CIENTÍFICAS*
}

\author{
CULTURAL SPECIALITY AND SOCIAL STRUCTURE OF \\ SCIENTIFIC COMMUNITIES
}

\author{
ANA M. GONZÁLEZ RAMOS y TERESA GONZÁLEZ DE LA FE \\ Universidad de la Laguna
}

\author{
PALABRAS CLAVE ADICIONALES \\ ADDITIONAL KEYWORDS \\ Estudios sociales de la ciencia, Campos de inves- \\ tigación, Actividad científica, Especialidades, \\ Social Studies of Science, Research Fields, \\ Culturas científicas y tecnológicas. \\ Scientific Activity, Specialities, Scientific and \\ Technological Cultures.
}

RESUMEN. Los estudios sociales sobre la ciencia han abordado de distintas maneras el trabajo realizado por las comunidades científicas refiriéndose a las características de sus miembros. Sin embargo, aún no se han determinado suficientemente los procesos de configuración social de la actividad científica. Por una parte, los límites entre las especialidades son difusos y crecen conforme lo hace su grado de especialización tecnológica y epistemológica. Por otra parte, eso se aborda de manera aislada en algunos campos del conocimiento pertenecientes a las "ciencias duras", de modo que tenemos un mosaico de descripciones diferentes, pero no una explicación global del modo de operar en ciencia en cualquier área de trabajo. A ello hay que añadir los cambios organizativos sufridos en el mundo de la ciencia en las últimas décadas, que inciden en los objetivos de los investigadores y en el contexto de la producción científica. Aquí argumentamos que el análisis de la estructura social de las comunidades científicas debe tener en cuenta las dimensiones epistemológicas, organizativas e, incluso, metodológicas desarrolladas por los científicos. Así, creemos que la práctica científica y la gestión de las tareas dentro de los equipos de investigación debem ser analizadas de manera global.

ABSTRACT. Social studies of science have dealt with the scientific communities from different viewpoints. However, it has been impossible hitherto to determine the social processes of scientific activity configuration. On the one hand, the limits of the specialities remain unclear and this uncertainty increases as the technological and epistemological processess become more complicated. On the other hand, these studies get on to some specialities of "hard sciences", so that we have a mosaic of different descriptions but not a global explanation of the operation modes in science, in any area of work. In addition, organizational changes in sciencie during the last decades, derive in the modifications of the researches objetives and the contexts of the scientific production. We argue here, that the analysis of the social structure of the scientific community must take into account the epistemological, organizational and indeed, methodological dimensions developed by the scientists. Thus, it must be approached in a global way, the scientific practices and task management within the research teams.

"Este trabajo ha sido posible gracias a la financiación del proyecto SCITECAN "Análisis sociológico y modelo computacional del sistema de ciencia, tecnología e innovación de las Islas Canarias" PI2003/173, BOC de Canarias 31, 16/2/2004

E-mail: amgonzal@ull.es tgdelafe@ull.es

Revista Internacional de Sociología (RIS)

Tercera Época, 42, Septiembre-Diciembre, 2005, 39-67 
RIS

REVISTA INTERNACIONAL DE SOCIOLOGIA

№ 42, SEPTIEMBRE-DICLIEMBRE, 2005

ANA M. GONZÁLEZ RAMOS Y TERESA GONZÁLEZ DE LA FE

\section{INTRODUCCIÓN}

El objetivo de este trabajo responde a la necesidad de sistematizar ciertos aspectos que evidencian el efecto transversal de la cultura sobre la actividad científica. La cultura científica afecta y matiza de manera muy sutil la labor de los investigadores, dejando una impronta específica según sus áreas de conocimiento de pertenencia. Normalmente asumimos la existencia de comportamientos distintivos en cada especialidad del conocimiento, dando por hecho que cada una de ellas cuenta con unos rasgos característicos y un conjunto de valores que influyen tanto en sus miembros como en sus instituciones de referencia (Merton, 1973; Becher, 1989). Pero estas consideraciones no están construidas sobre bases sólidas, existiendo numerosas lagunas, relacionadas incluso con la capacidad de delimitar las fronteras de cada especialidad científica. Así, se ha ido construyendo una serie de estereotipos que tienen que ver con lo que se espera de cada una de las disciplinas, sin importarnos demasiado la exactitud de esas valoraciones. Por eso, es preciso profundizar en las pautas de trabajo de los científicos, con el fin de comparar las distintas culturas de las distintas áreas de conocimiento. De este modo, profundizaremos en el conocimiento de la singularidad de la actividad científica, al mismo tiempo que en las diferencias existentes entre grandes ramas de la ciencia.

Un hecho social se considera relevante cuando se desprenden de él una serie de consecuencias que afectan a un conjunto de fenómenos sobre los que influye concatenadamente. De la misma manera, la variable cultura es relevante en el estudio de las prácticas científicas porque su influencia se estima decisiva en los procesos de investigación, en los resultados aportados por las distintas disciplinas y en la definición de los modelos de producción científica. Es decir, las culturas científicas explican el comportamiento diferente de las áreas de la ciencia (sociales, humanas, naturales, básicas, aplicadas o técnicas) y el que sus investigadores actúen de forma distinta en relación con su pertenencia a un grupo profesional determinado. En este estudio mostraremos cómo cada especialidad, más aún, cada subdisciplina, crea un espacio propio de referencia y unas pautas de comportamiento distintivas, que aportan entidad social al trabajo individual de los científicos. El procedimiento de recogida de información consiste en el examen sistemático de un conjunto de criterios relacionados con la actividad investigadora realizada desde distintos campos del conocimiento. De este modo, podemos establecer semejanzas y diferencias entre disciplinas y descubrir, desde esta perspectiva, los puntos comunes de la actividad científica.

Pero antes de continuar, es necesario reflexionar sobre la naturaleza de la ciencia y mostrar nuestra posición al respecto, ya que ésta influirá en la elección de los argumentos en los que apoyamos nuestra tesis. Algunos científicos actúan como si hubiese varios modelos de ciencia dependiendo de cada área científica. Bajo este supuesto, las distintas especialidades serían, por sí mismas, distintas 
maneras de observar el mundo, independientes entre sí y con metodologías de trabajo diferentes, es decir, varias ciencias. Nosotros, en cambio, consideramos que el método científico es único y que, además, lo es necesariamente, pues sus elementos de seguridad nos aportan garantías sobre la obtención de un conocimiento riguroso y seguro (el más riguroso y seguro posible desde un punto de vista lógico y racional; el mejor método de conocimiento hasta el momento). Esas garantías y procedimientos normativos deben ser perseguidos en cualquier disciplina científica, al margen de que cada una de ellas pueda hacer un uso diferente de los métodos de trabajo y de las técnicas de investigación, pero no de los criterios que sostienen al "método científico". La necesidad de utilizar diferentes técnicas se deriva de la consideración específica que los campos del conocimiento hacen de sus propios objetos de estudio, pero sin transgredir los principios básicos del método científico. Un ejemplo nos ayudará a aclarar esta idea: aunque un biólogo y un geólogo estudien el mismo campo del conocimiento, uno observará los seres vivos, y otro sus sustancias materiales; por ello utilizarán distintas técnicas y distintos procedimientos básicos, pero todos esos instrumentos responden a los criterios de objetividad, fiabilidad, validez y racionalidad del método científico. Podemos utilizar una metáfora lingüística para describir este fenómeno: todos los científicos utilizan el mismo lenguaje de comprensión de la realidad a pesar de que cada uno de ellos emplee idiomas distintos para abordarla.

Ciertas técnicas de investigación se emplean más frecuentemente en unas especialidades que en otras, aunque no exclusivamente en una sola de ellas de manera característica, es decir, puntualizando ciertos rasgos de esa herramienta de investigación. Esta es, precisamente, una de las hipótesis a prueba: los investigadores adoptan las técnicas más por la costumbre ${ }^{1}$ que por otras razones indudablemente relacionadas con sus fundamentos científicos. Los experimentos, por ejemplo, son más utilizados por psicólogos, médicos o biólogos, pero casi nunca por sociólogos; las fuentes orales, por historiadores y antropólogos, y casi nunca por químicos. Nuestra postura es que el objeto de estudio determina, a priori, esa elección de procedimientos, pero la cultura y la experiencia que se tienen de esa técnica definen en gran medida también la elección. Posteriormente, cada campo del conocimiento desarrolla de una manera peculiar la utilización de esas técnicas, reelaborándolas según sus necesidades, poniéndoles un acento particular propio de esa subdisciplina (Wray, 2005).

La idea de que existe un tipo de ciencia por cada una de las disciplinas científicas, además de absurda, pone en entredicho la naturaleza del método científico, amenazando los criterios que nos aportan seguridad como método seguro del

\footnotetext{
${ }^{1}$ Costumbre aquí se refiere a la experiencia previa, es decir, el conocimiento que se tiene de cada procedimiento de trabajo y que, por eso mismo, se convierte en el método más eficaz de utilización durante el proceso de investigación.
} 
conocimiento. Supone vía libre para utilizar los métodos según nuestro parecer y abre la puerta al incremento de errores provocados por el investigador. Si ignoramos los controles que corresponden a cada metodología, corremos el riesgo de considerar válidos unos resultados sesgados. En ocasiones, se desprecian los criterios de seguridad del método científico por corresponder con el modelo clásico de conocimiento de las ciencias naturales: cuantitativo y matematizado (aunque es falaz que este modelo pertenezca exclusivamente a un solo campo del conocimiento). La atención cuidadosa de los procedimientos con los que ejecutamos cada operación científica y la consideración previa de cuáles son los posibles sesgos que hayamos podido cometer en el estudio, debe ser una operación común y habitual a cualquier técnica y método de investigación. Como han demostrado King et al (2000), la utilización de una u otra metodología no exime a los científicos de mostrar, junto a sus resultados, los mecanismos de seguridad que los avalan, demostrando así que se han utilizado todos los medios a su alcance para aportar la máxima calidad a sus conclusiones.

Antes de finalizar este epígrafe introductorio, hay que hacer dos consideraciones adicionales. La primera se refiere a la utilización de la palabra ciencia, que en este trabajo se emplea en un sentido amplio, entendiendo por ella cualquier disciplina incluida en los programas de educación superior, independientemente del contexto donde se realice y del tipo de resultados derivados de su trabajo (académicos, de mercado, básicos o aplicados, filosóficos o empíricos...), incluso del tamaño de ese círculo social (Crane, 1972) o de si constituye una rama específica o tan sólo una subdisciplina dentro de un área más general. Así, hablaremos de disciplinas como el derecho, que está desprovisto de una base empírica y es estrictamente normativo, pero que, en el lenguaje cotidiano, es considerada de este modo inequívocamente y que, por la organización social de ese colectivo dentro del ámbito académico, no se distingue esencialmente de cualquier otra disciplina. En segundo lugar, nuestra aclaración se refiere al concepto de comunidad científica, pues no ignoramos el debate de los autores clásicos en torno a esa terminología. El término comunidad se complementa con el de sociedad, ambos definidos por Tönies y Weber para referirse a elementos cercanos pero de distinta naturaleza. Ambos se refieren a la unión de un conjunto de individuos por voluntad de sus miembros, pero en el primero prevalece la voluntad natural, mientras que en el segundo prevalece la voluntad reflexiva. La confusión terminológica comienza con el hecho de que ambos son modelos puros, lo que difículta su correspondencia con elementos de la realidad de manera inequívoca. Además, Tönies enlaza la voluntad reflexiva con la causalidad científica y, por tanto, con la producción científica. Sin embargo, este término no es aplicable a la naturaleza de las relaciones entre sus miembros; en ese caso se les reservaría el concepto de comunidad. De ahí surge el debate sobre la validez de la utilización de este concepto al referirse a la sociedad conformada por los científicos, ya que por la propia naturaleza de su tarea debería regirse por el principio de racionalidad; pero al referirse a las relaciones 
entre científicos sería más adecuado emplear el concepto de comunidad, puesto que estarían enlazados por vínculos personales. Esto es una prueba de que se corresponden con modelos ideales difícilmente identificables en la realidad. Tal como lo utilizamos aquí, la acepción más cercana a nuestro plantemiento general sería el término de comunidad, pues tratamos, precisamente, de mostrar cuáles son los elementos no racionales de la actividad científica y el modo de organización no formal que se produce dentro de su labor cotidiana.

La estructura de este trabajo mostrará, en primer lugar, el modo en que evolucionan las disciplinas científicas a medida que se emplean herramientas tecnológicas más sofisticadas y sus objetos de estudio se hacen cada vez más complejos, entrelazándose con ramas afínes en respuesta a la necesidad de contemplar de manera diferente la realidad (Wray, 2005). Ello también supone una división de las ciencias en relación con cada momento histórico y se relaciona con la aceptación de una serie de prácticas y valores que conforman la cultura de ese grupo social en ese momento dado (Merton, 2002: 627). En segundo lugar, se revisan aquellas variables relacionadas con las características demográficas y sociales de los agentes productores del conocimiento científico, lo que condiciona su modo de operar en la ciencia. A este planteamiento clásico hemos añadido otras dimensiones que tienen que ver con la modificación de las estructuras organizativas en el mundo de la ciencia (del Modo 1 al Modo 2), pero también, de una manera más general, con los cambios producidos debido a la necesidad de buscar recursos en un contexto de escasez o de contemplar la producción científica de una manera más aplicada y útil para la sociedad. En tercer lugar, nos ocupamos del elemento más original de este trabajo, cual es el análisis de las metodologías científicas empleadas por los investigadores, teniendo en cuenta su división por disciplinas, así como la estrategia que siguen para elegir sus líneas de trabajo y el diseño de sus investigaciones. También en esta ocasión tendremos en cuenta cómo les afectan las transformaciones que se están sucediendo en el contexto académico y científico.

\section{NATURALEZA DE LAS DISCIPLINAS CIENTÍFICAS}

La literatura está de acuerdo en señalar que la actividad científica es gremial y colectiva, pero, a partir de este punto, sus matizaciones acerca de lo que se entiende por comunidad científica son muy diferentes entre sí. Kuhn (1977: 16), por ejemplo, afirma que a los científicos se les reconoce descubriendo sus pautas de educación y comunicación, evocando de este modo el proceso de socialización que se produce durante la etapa de formación de los futuros miembros, en la que aprenden las normas y el funcionamiento del grupo. La definición aportada por Becher (1989: 20) nos lleva más allá, pretendiendo que se produce desde el mismo momento inicial de constitución de una disciplina científica, cuando se 
establecen sus bases epistemológicas específicas. Bajo este punto de vista, los rasgos culturales de cada rama científica estarían delimitados por la conceptualización que la comunidad científica hace de "su" ciencia y de la manera en que haya establecido los principios fundamentales de su objeto de estudio. Esta idea, expresada de diversos modos, es la opinión más generalizada en la comunidad científica, pero aceptarla supone asumir una serie de concepciones problemáticas. La primera es que no aclara el origen de esa toma de decisiones inicial, ni expresa las condiciones imperantes, es decir, los valores que pudieron filtrarse durante la "fundación" de esa disciplina. En segundo lugar, porque ignora los procesos de cambio que se producen en las ciencias y que originan las divisiones que darán lugar a las subdisciplinas, o también que modifican con el transcurso del tiempo la propia disciplina al ofrecer una orientación diferente de sus objetivos, métodos o procedimientos.

Precisamente porque las fronteras entre disciplinas se vuelven difusas y porque están sujetas a los vaivenes del tiempo, la distinción clásica entre áreas de conocimiento es compleja y queda sujeta a los elementos particulares que se quieran destacar de su actividad científica general. Las clasificaciones propuestas utilizan varios criterios de definición, que van desde la atención a su objeto de estudio, al empleo de una metodología específica e, incluso, a la manera en que la cultura ha institucionalizado su peculiar forma de enfrentarse a su campo de conocimiento. Storer (1976: 79) estableció la delimitación quizá más famosa, la que las divide en ciencias duras y blandas, dependiendo de su grado de rigor (derivado del uso más extensivo de las matemáticas en las primeras) y la impersonalidad (de nuevo, a favor de las ciencias duras) que ofrece un mecanismo de protección a sus miembros ${ }^{2}$. Kuhn (1977: 32-34), haciendo alarde de su valiosa experiencia como científico que ha ejercido en varios campos del conocimiento, aporta una descripción casi etnográfica de esa separación entre áreas. Asegura que cuando un físico presenta su trabajo final, las pruebas concluyen por sí mismas toda su aportación analítica. El trabajo de recogida de datos de un historiador y la manera en que los datos se vuelven relevantes desde un punto de vista comprensivo requiere un proceso más prolongado y laborioso, que conlleva sucesivas revisiones sobre los hechos. Finalmente, sobre la filosofia, afirma que nunca puede darse por concluida, pues ninguno de sus resultados son definitivos y su aportación al conocimiento está sometida a la continua reelaboración crítica de

\footnotetext{
${ }^{2}$ En efecto, Storer dice que el método matemático y el factor impersonal de las ciencias permiten a los investigadores ver disminuida su responsabilidad sobre los resultados encontrados. Es curioso que también Gaston de Bachelard, en otro contexto, explica que "la ciencia del solitario es cualitativa" mientras que "la ciencia socializada es cuantitativa" (2000: 285), un hecho que hoy en día es cada vez menos común, pero que no deja de reflejar esa relación complementaria entre matematización (o, mejor dicho, utilización de la estadística) y ciencia producida en equipo.
} 
otros filósofos y teorías elaboradas posteriormente. Becher (1989: 16) afirma que cada campo disciplinar (o grupos de especialistas) se define por las tareas intelectuales desempeñadas, alargando el glosario de ellas. Todas las nomenclaturas que podamos reunir (la división entre ciencias duras o blandas, sociales y humanas frente a físicas y naturales, aplicadas o básicas, técnicas o científicas ${ }^{3}$, incluso otras más específicas detallando cada especialidad y subespecialidad) se fundamentan en un aspecto concreto del trabajo que realizan las ciencias y las sitúan frente a otras especialidades científicas. Es decir, las clasificaciones sobre las ciencias se elaboran en base a la oposición entre ellas, lo que permite establecer sus rasgos identitarios. Las motivaciones epistemológicas esgrimidas por los padres de la disciplina para argumentar la necesidad de su constitución pueden volverse dudosas si observamos los entresijos de su delimitación, es decir, cuando, por ejemplo, la disciplina bebe de varias tradiciones, como ocurre hoy en día con la neurobiología, que se nutre tanto de la medicina como de las matemáticas, la física o la psicología, o cuando sufre un cambio de orientación que remueve los fundamentos originales de la disciplina, como el que protagonizan las ciencias de la computación actualmente. Sin embargo, la tradición y la manera de comportarse de sus miembros hacen evidentes, sin lugar a duda, casi desde el principio ${ }^{4}$, a qué especialidad pertenece. Parece existir un núcleo duro creado a partir de una serie de elementos difusos, pero significativos, sobre los cuales se crea la identidad de ese colectivo y que se mantiene a pesar de los cambios acaecidos en su seno, impidiendo la confusión entre un biólogo y un químico a pesar de las similitudes que puede haber entre ellos y encajando, sin embargo, dentro de la misma categoría a un biólogo del siglo XVIII y a uno del siglo XXI a pesar de la diferencia existente entre sus métodos y, sobre todo, a la transformación sufrida por su objeto de estudio.

El nacimiento de una disciplina suele coincidir con un hecho (o conjunto de ellos) especialmente relevante. Se establece, por ejemplo, que la cátedra de semiótica de la Universidad de Bolonia ocupada por Umberto Eco es el punto de partida de esa nueva disciplina, aunque esa palabra ya fuera utilizada anteriormente por Peirce y Saussure. Está ligado a elementos fundamentales del proceso de creación de una comunidad, como el reclutamiento de nuevos miembros o

\footnotetext{
${ }^{3}$ Becher (1989), citando a Dasgupta y David (1985), define a los científicos como aquellos profesionales que consideran el conocimiento como un bien de consumo público, mientras que los tecnólogos considerarían el conocimiento como un bien de capital privado, con estrategias relacionadas con la revelación y el secreto.

${ }^{4}$ Esto es así incluso para disciplinas como la sociología, cuyo nacimiento es tan discutido como cabe esperar en una disciplina autoreferente. En su nacimiento, Comte vertió sus críticas sobre aquellos "contadores de fenómenos sociales" como Quetelet. Sus opiniones, avaladas por su reputación, suponían un modo correcto de proceder en sociología. Decantarse por la contabilización de fenómenos hubiera orientado a esta displina hacia otros derroteros.
} 
RIS

la creación de una revista especializada sobre el área de conocimiento, lo que supone al mismo tiempo un canal de información y el establecimiento de un mecanismo de distribución de recompensas académicas. Supone en todos los casos un ejercicio de poder de un colectivo que apuesta definitivamente por un cuerpo de conocimientos especializados. Desde ese punto de vista, puede decirse que el nacimiento de una especialidad científica es un hecho construido socialmente, fruto de un acto de voluntad por el que se define "políticamente" el sentido y la intención de esa disciplina que se apropia de un área de problemas concreta (Mulkay, 1977), sobre el que se adopta un enfoque característico y unos métodos apropiados (Whitley, 1974). Pero su evolución no termina ahí; la ciencia seguirá nutriéndose a lo largo del tiempo de las aportaciones elaboradas por otros campos afines. Sin embargo, será más vulnerable a las influencias en las primeras fases de su composición, cuando los pioneros tienen más probabilidad de proceder de otras ramas y de "contaminarlas" con elementos externos útiles a su planteamiento inicial (Wray, 2005). A medida que las aportaciones se hacen más independientes y específicas, su metodología y contenidos se revelan como rasgos identitarios de esa disciplina y la marcarán para siempre. Así sucede, por ejemplo, con la alquimia, disciplina ya desaparecida, pero que podemos vincular con la química y la farmacia modernas (Hankins, 1988; Bacherlard, 2000), ciencias en las que podemos encontrar aún las huellas de la primera, una disciplina básicamente experimental. Por supuesto, ni las herramientas ni los objetivos son ya los mismos, pero permanecen ciertas ideas generales que daban sentido a la práctica de la alquimia: la experimentación en el laboratorio con unas sustancias a partir de las cuales hay que sintetizar otras más complejas; por no hablar de otros fundamentos más discutibles, de carácter filosófico, como la búsqueda de un remedio contra cualquier mal, que podría asociarse con la búsqueda de la piedra filosofal. La medicina puede servirnos de segundo ejemplo para demostrar esa mayor vulnerabilidad de las ciencias en los albores de su nacimiento. Su desarrollo ha ido aparejado siempre a la propia existencia biológica de los seres vivos, pero en ese momento no puede considerarse conocimiento científico, pues se trataba, sobre todo, de desempeñar una función curativa que sólo se corresponde con una parte del cuerpo de conocimientos de la medicina actual o que, incluso, nos remitiría al campo de la enfermería. Su (re)fundación científica en la historia moderna está estrechamente relacionada con los adelantos científicos y técnicos aportados por otros campos del conocimiento: la óptica le proporcionó su conocimiento sobre los cristales dando lugar a la microbiología; el arte sus conocimientos anatómicos y los modelos de utilidad sobre el cuerpo humano; la estadística fue fundamental en la investigación de las principales tendencias epidemiológicas (López Piñero, 2000). A pesar del gran número de especialidades y variedad de prácticas que conlleva la ciencia médica, reconocemos a cada miembro de ese colectivo con la misma denominación, separándolos unívocamente de otras profesiones paralelas como el fisioterapeuta, el farmacéutico y otras tantas. 
El proceso de cristalización de una ciencia o especialidad científica nos ofrece la oportunidad de comprender la naturaleza de esa ciencia, pero Bourdieu (2003) señala otros factores relacionados con el proceso de socialización, como característica de delimitación de la actividad científica. Según él (Bourdieu, 2003: 76), el "oficio de científico" proviene de su origen social y de sus características de género, sus trayectorias profesionales y la posición ocupada dentro el sistema. Los rasgos disciplinarios son, sin duda, los de mayor envergadura, es decir, el hábito que se desprende del desempeño de los roles ordinarios en cada uno de esos niveles de la estructura social. La asunción de las funciones sociales y los principios generales de una disciplina permiten a un científico asumir como suya la sabiduría de otros científicos que se preguntaron por esas mismas cuestiones anteriormente, $\mathrm{y}$ que destacaron por la superioridad de sus aportaciones y la validez de sus resultados. Bachelard plantea una explicación similar ${ }^{5}$ cuando denomina a la época precientífica como una etapa de infancia de la ciencia, pues las explicaciones ofrecidas por los científicos de salón (como se les denomina para hacer hincapié en el escaso o precario trabajo de laboratorio que desarrollaban) contienen una gran dosis de inocencia, tanto es así, que muchos de sus razonamientos "científicos" sólo adquieren la categoría de broma. Sus repuestas a los problemas científicos están llenos de prejuicios y de irracionalidades, originadas, en parte, por la teocracia vigente (Bachelard, 2000: 30-62), pero también por la ingenuidad de sus planteamientos. Algunos de los ejemplos que ofrece Bachelard para mostrar el candor de sus respuestas son el abuso de las metáforas y su continua referencia a procesos animistas para describir fenómenos naturales como la indigestión o la inflamabilidad de los cuerpos. Una prueba de lo común que era esa postura en esa época es el hecho de que se vieran implicados incluso personajes que hoy se consideran grandes pensadores de su tiempo, como la superstición de la que se vale Bacon para explicar cómo podemos librarnos de las verrugas (2000: 173). La suficiencia de los contenidos explicativos y la aportación de un buen número de pruebas sobre el que hacer recaer la explicación teórica no llegará hasta que se alcance la madurez científica; mientras tanto, sus simplificaciones sobre la naturaleza podrían atribuirse a niños de ocho años de nuestro tiempo (2000: 44). Bourdieu retoma esta idea, de una manera más indulgente, poniendo de manifiesto que un matemático de veinte años en la actualidad puede tener en su haber veinte años de matemáticas, es decir, el conocimiento recopilado durante más de veinte años durante los cuales sus predecesores han aportado su experiencia, éxitos y fracasos, y que han reparado muchas de las ingenuidades que supusieron el primer acercamiento a esa serie de problemas. La inexcusable necesidad de acumulación y superación de las ciencias hace que la socialización (obligada en

\footnotetext{
${ }^{5}$ Aunque Bourdieu no menciona en ningún momento a Bachelard es posible establecer un nexo de unión entre los dos autores franceses.
} 
cualquier actividad social) tenga un significado mucho más profundo en la actividad científica. La socialización de un científico supone la asimilación de valores y conocimientos que deben darse por sentado, sin discusión casi por necesidad, ya que permite el imparable proceso de descubrimiento científico.

Para Kuhn (1977) el consenso es el elemento que da consistencia a la comunidad de científicos, un requisito indispensable a partir del cual se establece el conjunto de problemas conceptuales seleccionados por ese campo del conocimiento e indispensable para permitir el progreso de la ciencia, pues ejerce una función legitimadora. En algunos casos, la aceptación de los hechos que serán pertinentes para una corriente científica en un momento dado, puede deberse más a la legitimidad que aporta el principio de autoridad que a la existencia de un número suficiente de evidencias empíricas ${ }^{6}$. Por otra parte, la presión que ejerce la cultura del grupo sobre sus miembros es imprescindible para reforzar su identidad del grupo, por lo que es probable que de ahí procedan las "batallas" entre disciplinas científicas distintas (la biología con la medicina o la sociología con la psicología, por ejemplo, donde la diferenciación respecto a los otros campos del conocimiento supone la reivindicación del nosotros). No por casualidad esas confrontaciones se producen, en mayor medida, entre aquellas ramas que tienen más puntos en común o cuyos planos conceptuales se superponen. También existe un acuerdo generalizado respecto al importantísimo papel que juega la "cultura" en la conformación de las distintas disciplinas científicas. Así, Merton (1973: 268) establece que la ciencia denota 1) un conjunto de métodos característicos, 2) una acumulación de conocimientos fruto de la aplicación de estos métodos, 3) una serie de valores culturales y costumbres que gobiernan las actividades científicas y 4) cualquier combinación de esos factores, lo que la convierte en una institución.

\section{DIFERENCIAS CULTURALES EN EL SENO DE LAS COMUNIDADES CIENTÍFICAS}

Bourdieu (2003) afirma que el tipo de ciencia elaborada por los científicos depende de sus características demográficas y sociales. Factores como la edad, la raza, los estilos de trabajo, el prestigio y la nacionalidad de los científicos han formado parte del conjunto de variables analizadas en numerosos estudios empíricos para conocer su influencia sobre la actividad científica. Entre ellas, la edad parece ser un factor especialmente decisivo (Torres, 1989: 117), más que otros rasgos influyentes en la actividad científica (González Ramos, 2004), pues es especialmente

\footnotetext{
${ }^{6}$ Hay suficientes ejemplos en la historia de las ciencias para avalar estas palabras. Podemos evocar, por ejemplo, el relato que hace Koyré (1981) sobre los primeros pasos de la física.
} 
sensible a los cambios de paradigmas y al avance de las técnicas de investigación. La juventud de los investigadores proporciona, según Merton, una base para el cambio científico (1973: 510-514), pues aporta mayores probabilidades de cambio mediante la innovación y la generación de nuevas ideas. Los jóvenes tienen mayor predisposición a adaptarse a las transformaciones y exigencias que imponen los contextos externos y la estructura normativa de la ciencia (Sedaitis, 2000; Bozeman y Mansematin, 2004). En este mismo sentido, los mecanismos a través de los cuales se ponen en marcha las estrategias de competitividad científica serían más rápidamente incorporados por los jóvenes que por los científicos más veteranos (Becher, 1989: 167; Bijker, 1997: 126; González Ramos, 2004: 98). En varios estudios (Bozeman y Mansematin, 2004; González Ramos, 2004) se ha encontrado que la edad es una variable decisiva para determinar cuál es el estilo de trabajo adoptado por los científicos. Los investigadores noveles parecen ser los primeros en ajustarse a la forma de trabajo auspiciado bajo el Modo 2 (Gibbons et al, 1997). Por ejemplo, en el estudio de casos realizado sobre la comunidad científica de la Universidad de Cádiz (González Ramos, 2004), los artículos escritos por autores jóvenes tienen una mayor probabilidad de estar elaborados en colaboración con investigadores de otras universidades extranjeras o pertenecientes a centros de investigación no académicos, tal y como se espera que ocurra en la ciencia post-académica. Sus contribuciones también reportan al conjunto de la universidad una mayor relevancia en cuanto a la cantidad de artículos e índices de referencia acumulados y representan, por tanto, una ventaja competitiva para esa institución.

Otras variables, como el género de los investigadores, no presentan evidencias tan relevantes en cuanto a la influencia que ejercen en el modelo de trabajo de los científicos en relación con su actividad investigadora. Las mujeres sufren numerosas dificultades, relacionadas, especialmente, con su acceso en términos de igualdad a la estructura profesional, pero sobre el ejercicio de su función investigadora sólo puede decirse que su actividad es similar a la de sus compañeros de trabajo (Long y Fox, 1995). En el estudio de González Ramos (2004) podemos comprobar el efecto que provocan estas dos variables, sexo y edad, en la actividad de los investigadores: mientras la edad supone un elemento de diferenciación de las pautas de comportamiento halladas entre los científicos, el género no condiciona el resultado de su trabajo, es decir, el número e importancia de sus índices de impacto. Las mujeres químicas obtienen tasas de productividad científica similares a los de sus compañeros masculinos y, a su vez, diferentes a las de las mujeres dedicadas a la investigación pertenecientes a otras ramas científicas como la filología. Las diferencias entre la productividad de hombres y mujeres se deben al comportamiento distintivo de las áreas de conocimiento, pero no a su género, pues el comportamiento de ambos grupos es similar dentro de cada disciplina y puede decirse definitivamente que no está causada por dicha característica. En contraposición, el modelo de conducta puede ser distinto entre personas pertenecientes a distintas generaciones o a distintas áreas de conocimiento. 
RIS

REVISTA INTERNACIONAL DE SOCIOLOCIA

Los estudios biográficos de mujeres científicas nos aportan argumentos similares y, probablemente, más reveladores. En Barral et al, (1999: 291-324) encontramos el discurso de cuatro científicas (del campo de la biología, la medicina y la física y procedentes de ámbitos académicos y de la empresa privada) que relatan sus trayectorias familiares, profesionales y, en definitiva, su propia experiencia en el mundo de la ciencia. Sus discursos denotan una clara aceptación de su condición profesional independientemente del hecho de ser mujer o no. Hablan, sobre todo, de una España atrasada en materia educativa (hay que tener en cuenta la edad de las informantes) y, aunque no dejan de referirse a las dificultades sociales con las que se encontraron, principalmente relacionadas con las condiciones desiguales de acceso al empleo o la dificultad de llegar a puestos de relevancia dentro de su esfera profesional, sus opiniones no denotan un sentimiento o conciencia de desigualdad respecto a otros asuntos relacionados con su forma de trabajo. Sus palabras no revelan que se consideren con un estatus distinto al de sus compañeros ni que su actividad científica difiera de la de sus compañeros masculinos ${ }^{7}$. La dificultad estriba en la lucha por el empleo y en las oportunidades de obtener un lugar estratégico dentro de su carrera profesional, pero en cuanto a su actividad no ofrecen opiniones que revelen la existencia de comportamientos distintivos (a este respecto, véase también Long y Fox, 1995). Sin embargo, cuando atendemos a los discursos de las investigadoras de ciencias humanas, mucho más sensibles a las corrientes feministas, la percepción cambia diametralmente. En el mismo libro (Barral et al, 1999) otras autoras (Sánchez González, 1999: 259-290 y Alemany, 1999: 81-99) hablan de la ocultación que ha sufrido históricamente el trabajo de las científicas o de cómo la tecnología se hace a espaldas de las mujeres a pesar de ser ellas las principales usuarias (como en el caso de los electrodomésticos). Estos argumentos, ciertos indudablemente, no invalidan los anteriores, que muestran cómo la productividad o el tipo de trabajo desarrollados por las científicas es similar al de los científicos, siendo el motivo por el cual las mujeres se sienten iguales a sus compañeros en lo que se refiere a su actividad científica. Este es por sí mismo, en nuestra opinión, un indefectible rasgo de igualdad.

En el trasfondo de estas argumentaciones se encuentra nuevamente la pertenencia a un área de conocimiento, lo que supone un modo de trabajo distinto para los investigadores. Cada disciplina tiene un objeto de estudio y una finalidad concreta para realizar su trabajo, en razón a los cuales se emplean distintos métodos de trabajo (por ejemplo, distintas maneras de relacionarse con sus colegas de profesión). Así, el tipo de documento preferido por las ciencias naturales es

\footnotetext{
${ }^{7}$ Son especialmente claras en este sentido, las palabras de Teresa Rodrigo, doctora en física de la Universidad de Cantabria, y M. Jesús Azanza, bióloga de la Universidad de Navarra, (pp. 311 y 319 respectivamente).
} 
el artículo, mientras en las ciencias sociales se prefiere la modalidad de libro, probablemente porque el informe es más idóneo para expresar los resultados globales producidos por sus estudios. Los artículos escritos por los científicos naturales son firmados por más de un autor, de modo que queda claro, expresamente, que es la consecuencia de un trabajo colectivo (quizá, siguiendo a Storer, la costumbre provenga de la estrategia de protección de los miembros del grupo), mientras que los científicos sociales frecuentemente escriben artículos en solitario. Merton (1973) destacó la función de promoción que proporciona la autoría colectiva, pues combina los esfuerzos renovados de los autores noveles, con el prestigio y la experiencia de los más veteranos. Sin embargo, este procedimiento es más típico de las ciencias naturales que de las ciencias sociales y humanas, donde se utiliza en menor medida, problablemente, como reflejo de su modo de trabajo individualizado, es decir, que no cuenta con un equipo de trabajo estable y duradero ${ }^{8}$. Merton (1973: 552) relacionó el fenómeno de la multiautoría con la transformación de la ciencia (de la Little Science a la Big Science), proceso que dió lugar a un nuevo modo de considerar la ciencia imponiendo ciertas normas de obligado cumplimiento, que fue creciendo paulatinamente a lo largo del siglo $\mathrm{XX}$ : primero, entre los investigadores de las ciencias naturales; posteriormente entre los sociales (Merton, 1938; 1973: 408-409). Esto parece sugerir que cada ciencia se adapta a los cambios normativos a un ritmo diferente, lo cual refuerza la idea de que la cultura del grupo ofrece una identidad particular a sus miembros, incluso a pesar de las transformaciones de su contexto. Otra diferencia esencial entre distintas disciplinas científicas radica en la proyección de sus logros; nos referimos al hecho de que los científicos naturales usualmente escriben en inglés y en revistas internacionales, mientras los científicos de ciencias sociales y humanidades lo hacen en mayor medida en español y en revistas de ámbito nacional. De este comportamiento se deduce una serie de consecuencias significativas respecto a la importancia que conceden a la difusión de su trabajo. El hecho de que los artículos pertenecientes al campo de las ciencias naturales se escriban en inglés, les reporta la oportunidad de publicar en revistas internacionales, ser leídos en el extranjero y ser reconocidos por sus aportaciones en otros países, además de aportar mayor fluidez a la información generada en su campo del conocimiento. Por el contrario, las ciencias sociales y humanas se restringen a un ámbito de referencia más limitado, a la esfera nacional como máximo. Ello explica (o así se expresan habitualmente los profesionales del derecho, la historia y la sociología, entre otros) porque los temas sobre los que versan sus objetos de estudio tienen un contexto de referencia más limitado (muy vinculados a la realidad geográfica

\footnotetext{
${ }^{8}$ Esta tendencia es más acentuada aún en las ciencias humanas, según González Ramos (2004: $75,196)$.
} 
RIS

REVISTA INTERNACIONAL DE SOCIOLOGÍA

№ 42, SEPTIEMBRE-DICIEMBRE, 2005

ANA M. GONZÁLEZ RAMOS Y TERESA GONZÁLEZ DE LA FE

de donde se extraen), mientras que los hechos sobre los que trabajan los científicos naturales atañen a funciones biológicas o físicas que son fenómenos generales, de carácter universal. Independientemente de cuál sea la razón por la que se actúa de esta manera y de la validez de sus argumentos, todo ello evidencia un estilo de trabajo diferente entre grandes áreas del conocimiento $\mathrm{y}$, posiblemente, una valoración distinta del modo y la finalidad de su trabajo. Los artículos parecen ser más adecuados para mostrar el tipo de trabajo llevado a cabo por los científicos físicos, con posibilidad de mostrar poco a poco las pruebas que van verificándose en el trabajo global realizado por todo el equipo. Los libros, en cambio, permitirían a los científicos sociales y humanos ser más prolijos al mostrar toda la serie de aspectos analizados en su investigación, que reflejan un realidad más extensa, referida a una comunidad o fenómeno social completo (González Ramos, 2004: 74).

Otra diferencia significativa es el grado de interdisciplinariedad y colaboración desarrollada por los grupos de investigación, de la cual suele decirse que la multidisciplinariedad es una práctica más asentada entre las ciencias naturales ${ }^{9}$. González Ramos (2004: 51-52) ha encontrado que los equipos de investigación de Derecho son los más homogéneos; para ello ha comprobado que sus equipos de investigación están compuestos casi exclusivamente por licenciados en derecho, pero de cerca le siguen los grupos de matemáticas y filología, por lo que no puede concluirse que sea un rasgo característico de las ciencias sociales o humanas. La composición de estos grupos tiene un alto grado de homogeneidad (de más del $40 \%$ atendiendo al origen curricular de sus miembros). En el otro extremo se sitúan las ramas tecnológicas, los más multidisciplinares, es decir, conformadas por especialistas de la misma área, pero distinta titulación o pertenecientes a distintas áreas y titulaciones. En este grupo estarían todas las ingenierías, ciencias agrícolas y medioambientales y ciencias de la salud. También el comportamiento de los equipos de investigación difiere de una rama del conocimiento a otra y entre disciplinas, en cuanto a su elección sobre el número y diversidad de temas establecidos como líneas de trabajo de sus programas de investigación. En el seno de muchos grupos del área de humanidades la actividad sigue siendo individual y el número total de líneas de trabajo del grupo es la suma del esfuerzo individual de los distintos investigadores, que se unirian para formar parte de un grupo de investigación con fines más bien normativos y administrativos ${ }^{10}$.

\footnotetext{
${ }^{9}$ Quizá este aspecto forme parte de un aspecto de carácter evolutivo, similar al descrito por Merton acerca de la multiautoría, puesto que la necesidad de constituir grupos con una morfología multidisciplinar está cada vez más presente en todas las disciplinas y áreas de conocimiento.

${ }^{10}$ Hay que recordar que alli donde hay una política científica que impone el registro y la medición de los grupos de investigación, se favorece la creación de grupos de trabajo y se fomenta, en general, el trabajo en equipo. Sin embargo, a veces, la unión de esos investigadores no tiene más que la función instrumental de concurrir a convocatorias oficiales de financiación, o beneficiarse de otras ventajas que le proporciona su estatus. Esto es una demostración, una vez más, de que la imposición normativa no siempre causa los efectos esperados, sobre todo si supone un cambio para el comportamiento habitual de los actores sociales.
} 
Las líneas abiertas en los grupos de humanidades son muy numerosas y bastante distantes entre sí, lo que denota un trabajo individualizado de los miembros del grupo, a pesar de su unión convencional dentro de un grupo de trabajo" ${ }^{11}$. Por el contrario, los grupos pertenecientes a las ciencias físico-naturales cuentan con varias líneas de trabajo de naturaleza similar, lo que refleja un trabajo conjunto, resultado de una labor colectiva y en equipo. Sin embargo, el trabajo individualizado dentro del grupo de investigación no es exclusivo de las áreas de humanidades, ya que también lo encontramos en los grupos de matemáticas, por ejemplo. Es posible que este comportamiento sea más habitual allí donde el grado de abstracción de la materia impida una fácil agrupación de los esfuerzos colectivos, por lo que el equipo se convierte en un instrumento administrativo, pero no en un instrumento de trabajo intelectual. En cambio, el trabajo colectivo de los equipos de investigación se produciría en mayor medida allí donde la parcelación en pequeñas secciones de la actividad investigadora puede lograr, conjuntamente, un resultado común. También puede deberse a la necesidad de contar con un alto grado de tecnificación, más costoso y/o más laborioso, que precisa una colaboración más estrecha entre los miembros del grupo. Esta misma argumentación explicaría la mayor multidisciplinariedad en los equipos de investigación de las áreas tecnológicas. Pero ninguna de estas hipótesis explicaría por qué algunos grupos del mismo campo (de matemáticas o de humanidades pongamos por caso), y previsiblemente con las mismas cotas de abstracción teórica, tipo de objeto de estudio y grado de tecnificación, realizarían un trabajo de equipo y multidisciplinar; mientras otros realizarían, uno individualizado y homogéneo. Quizá algunos tengan una visión más contemporánea del ejercicio de la actividad científica y otros sigan una praxis más tradicional respecto a su disciplina. En cualquier caso, debemos achacarlo a la cultura (o socialización científica), que define sus pautas de comportamiento habituales y les aporta un contenido concreto y separado de otros grupos de su propio campo (Campbell, 2003).

Becher (1989) plantea que el modo en que van adoptándose los diferentes modelos de trabajo en las diferentes disciplinas está relacionado con la manera en que se expresan los criterios de prestigio en sus respectivas culturas académicas ${ }^{12}$. El autor entrevista a científicos anglosajones pertenecientes a distintos campos del conocimiento y, a partir de sus opiniones, establece qué elementos define el reconocimiento de los pares en cada una de las distintas especialidades. Así, los historiadores y filólogos obtienen prestigio a medida que van publicando un mayor número de libros; en las ciencias "duras puras"y "duras aplicadas" (esto es,

\footnotetext{
"Estos datos se refieren al trabajo realizado por González Ramos (2004).

${ }^{12}$ El término "académicas" es utilizado aquí por ser el que utiliza Becher. Pero se refiere a la cultura particular de su segmento de trabajo, ya sea éste una institución académica superior, un departamento de investigación o cualquier otro espacio de producción científica $\mathrm{y}$, en todos los casos, supone un conjunto de elementos abstractos, pero efectivos, que definen el prestigio de una actividad científica y a su autor.
} 
física, química, ingeniería y farmacia) ${ }^{13}$ los libros serían escasos, como ya hemos visto, predominando los artículos científicos. En el caso de la sociología, muchos prefierirían la imagen del académico solitario porque implicaría distinguirse por encima de sus compañeros, según este autor, algo que aprendería durante la socialización de la fase de doctorado (Becher, 1989: 133). Pero sin duda, la manera más extendida y generalizada de valorar el trabajo de un científico se establece a través de la medición de la cantidad de citas recibidas por sus obras y por el prestigio de las revistas donde publican sus logros científicos. La importancia de esta dimensión ha cobrado una dimensión especial en los últimos tiempos, siendo cada vez más aceptada por los científicos como medida de evaluación, en parte por los cambios normativos sufridos en el sistema de ciencia y tecnología; sin embargo, también ha recibido numerosas y oportunas críticas. De cualquier modo, su estudio va mucho más allá de lo que se persigue en este trabajo, por lo que continuaremos con nuestro análisis, alejándonos de estos aspectos y abordando otros menos incómodos que, sin embargo, también se refieren al examen de las citas realizadas por un autor, pero en otro sentido distinto. En las ciencias naturales, toda cita bibliográfica empleada en un texto debe justificarse por su necesidad explicativa y debe corresponderse con una mención exclusiva del argumento ofrecido en el cuerpo de la argumentación, es decir, su mención es precisa para comprender los contenidos significativos del documento. En las ciencias sociales, en cambio, se hace una aplicación menos restrictiva, citando a autores relevantes no mencionados a lo largo del trabajo porque el autor considera que está próximo a la línea argumentativa del documento o, incluso, como mera señal de reconocimiento y de identificación con sus ideas. Entre los biólogos está claro que las citas deben ser un signo demostrativo de que se ha leído todo lo pertinente; en sociología las citas se utilizan para reforzar los planteamientos expresados, pero también para demostrar un mayor fundamento y para sugerir que tenemos buenos referentes intelectuales (Becher, 1989: 119-123).

El papel desempeñado por los becarios es otro rasgo de diferenciación cultural, una característica que denota una organización del trabajo diferente según las distintas especialidades científicas. Como han demostrado diversos trabajos (Fernández Esquinas, 2002; González de la Fe et al., 1996, entre otros), las pautas de comportamiento del personal en formación son muy distintas de un campo del conocimiento a otro. Uno de los factores más sorprendentes es el tiempo empleado para finalizar las tesis doctorales, según el cual el campo de la medicina sería la especialidad que emplearía menos tiempo, mientras que, por el contrario, las disciplinas integrantes en el núcleo de las ciencias sociales son las que mayor tiempo emplearían en cumplinar el proceso de elaboración

\footnotetext{
${ }^{13}$ Se utiliza aquí su propia nomenclatura, sumamente clarificadora dentro de este contexto. La cita proviene de la página 116.
} 
de tesis (Fernández Esquinas, 2002: 193). Del mismo modo, las tesis llevadas a cabo en los institutos de investigación tendrían una duración más corta y versarían acerca de temas más aplicados que las desarrolladas dentro de un contexto universitario, por lo que vemos que los modo operandi no sólo se refieren a las áreas del conocimiento, sino también a las instituciones donde se desarrolla el trabajo científico. Por otra parte, los grupos que requieren un trabajo de laboratorio para llevar a cabo sus investigaciones forman una red tupida entre maestros y aprendices que propicia los contactos periódicos entre el líder del grupo y sus doctorandos (Becher, 1989: 147). En el extremo opuesto se encontraría el doctorando que trabaja en solitario sobre un tema relativamente específico del que no puede esperarse el mismo grado de colaboración colectiva, pues él mismo, una vez profundizado en el tema, se convertirá en el máximo especialista sobre el tema. En tercer lugar, también es diferente el rol que ocupan los becarios según las distintas especialidades del conocimiento; en algunas de ellas es posible que un estudiante de doctorado tenga la misma responsabilidad en el trabajo que el resto de sus miembros (Becher, 1989: 147-148; González Ramos, 2004: 49, 90, 230) y, por ejemplo, pueda publicar un artículo en colaboración con sus profesores. En otras, esto no es posible simplemente porque se le considera personal en formación, insuficientemente maduro para realizar ciertas tareas que presuponen la capacidad investigadora. En definitiva, la socialización y las costumbres que van conformando la actividad científica de los investigadores, tanto entre los investigadores jóvenes como entre los más experimentados denotan, también una dinámica interna diferente en el seno de los grupos de investigación.

\section{USO DE LOS MÉTODOS CIENTÍFICOS Y ORIENTACIÓN DEL TRABAJO REALIZADO}

En este apartado se abordan dos aspectos relacionados: el primero se refiere al empleo de las técnicas de investigación en cada campo científico y, el segundo, a la orientación que empuja a los investigadores a seleccionar sus líneas de investigación principales. Respecto al primero de ellos, es preciso señalar que no existen trabajos anteriores sobre este asunto. La sociología de la ciencia ha analizado un importante número de aspectos referidos a la actividad científica de los investigadores, pero ninguno ha abordado el empleo de las técnicas de investigación que los científicos hacen en su actividad cotidiana. Por su parte, el campo de la metodología se ha encargado tradicionalemente de aspectos más técnicos, y el más cercano se refiere a los errores causados por las prácticas incorrectas de los investigadores durante la ejecución de la investigación o su diseño. Quienes están familiarizados con el mundo de la ciencia intuyen que en cada disciplina se emplea un conjunto de herramientas, utilizadas de una manera particular, pero sin tener datos empíricos que avalen esa opinión. Los trabajos empíricos que más se han 
acercado a este conjunto de cuestiones relevantes sobre la actividad científica de los investigadores han sido los estudios etnográficos realizados en el laboratorio. Con la intención de llenar este vacío, González Ramos (2004) ha emprendido un estudio que aborda el tema de una manera diferente, no desde una perspectiva etnográfica sino cuantitativa. Su objetivo es sistematizar la práctica habitual de los científicos para llegar a comprender globalmente este fenómeno.

Para ello ha diseñado una encuesta a través de la cual pregunta a los propios investigadores sobre sus procedimientos de trabajo más frecuentes, las técnicas de investigación que emplean usualmente y la manera en que las llevan a cabo con preguntas relativas tanto a su entorno de trabajo, como a los controles de seguridad que les permiten asegurar la calidad de sus resultados. Su estudio ha encontrado hallazgos significativos relacionados con la práctica cotidiana de los investigadores, constatando, por ejemplo, que la utilización de varias metodologías es habitual en cualquier grupo de investigación y que se usan las mismas técnicas de investigación independientemente del área de conocimiento a la que pertenezcan el equipo. De nuevo, la cultura de cada subdisciplina aparece como una fuente relevante de explicación, trascendiendo a aquellas opiniones que achacan la utilización de unas técnicas a las ciencias físicas y naturales y las otras, por oposición, a las ciencias sociales y humanas. Sin embargo, según este estudio, la utilización de un conjunto de herramientas de investigación no parece estar restringida a un solo grupo del conocimiento, ya que aproximadamente la mitad de los grupos utilizan frecuentemente varias técnicas en sus investigaciones. La utilización de una menor proporción de técnicas por parte de un mismo equipo de investigación se da más en aquellos que pertenecen a las áreas de tecnologías, (donde el 66,6\% de los grupos emplean una sola técnica de investigación), le siguen el campo de la salud $(62,5 \%)$ y el de ciencias sociales $(50 \%)^{14}$; el resto de las áreas conjugan más de una técnica de investigación en una alta proporción. Así pues, la mayoría de los grupos de humanidades, ciencias naturales, física, química y matemáticas utilizan normalmente varias técnicas de investigación.

Los diseños de investigación más habituales se basan en fuentes secundarias de información. La extensión de las fuentes secundarias como método de investigación más habitual entre los grupos puede deberse a la sobrerepresentación de los grupos de humanidades en esta universidad, pero, también, a la multiplicidad de contextos en los que puede aplicarse esta valiosa herramienta de recogida de datos. Como prueba de la complejidad del empleo de las técnicas de investigación por parte de los grupos de investigación, podemos destacar el hecho de que esta herramienta de investigación ni es utilizada por todos los grupos de humanidades de esta universidad ni únicamente por ellos, ya que otras áreas de conocimiento

\footnotetext{
${ }^{14}$ Esta alta proporción de utilización de una sola técnica en los grupos de ciencias sociales se debe al peso específico que tienen los grupos de derecho en esta área del conocimiento en el universo de estudio específico.
} 
como la biología y la medicina también la utilizan reiteradamente. Ello viene a demostrar que la utilización de las técnicas de investigación no está relacionada exclusivamente con el área del conocimiento al que se pertenece, sino a la naturaleza de las herramientas de investigación empleadas y a su utilidad para abordar un objeto de estudio. Así, la mayoría de los equipos de investigación describen su labor científica como una investigación cuantitativa, mientras que sólo el $35 \%$ de los grupos la calificaron de cualitativa. Consecuentemente, será muy frecuente el empleo de métodos de muestreo y el uso de programas informáticos para realizar sus tareas de recopilación y análisis de los datos. El 63\% de los grupos afirmaron que empleaban de manera habitual métodos de muestreo para llevar a cabo sus estudios y el $85 \%$ aseguraron que usaban programas informáticos para ayudarse en su trabajo cotidiano de investigación. A este respecto, no se produce ninguna distinción entre áreas de conocimiento, pues todas las especialidades cuentan con grupos que se apoyan en hojas de cálculo, programas específicos y bases de datos estadísticas para alcanzar sus resultados. Por tanto, puede concluirse que las herramientas de investigación no son exclusivas de una disciplina o conjunto de ellas, sino que se emplean en diferentes campos de la ciencia indistintamente. Ello apoya la tesis de que la división entre áreas, sobre todo si la simplificamos hasta el punto de diferenciar sólo entre ciencias naturales y ciencias sociales, ni es tan nítida ni exacta como parece, ni en relación a los límites que las dividen ni en las pautas de comportamiento y la utilización de las técnicas empleadas.

Sobre el objetivo que el grupo persigue con su trabajo mediante la elección de líneas y temas de investigación, hay que decir que la mayoría considera su trabajo predominantemente básico, ya que, a una pregunta indirecta relacionada con este asunto, respondieron que su trabajo contribuiría en mayor medida a "incrementar el conocimiento de su área" y a "mejorar las herramientas de estudio empleadas tradicionalmente". Sólo los equipos de ciencias sociales se mostraron más proclives a calificar su investigación de aplicada eligiendo, en la misma pregunta, otro ítem que reflejaba que su investigación contribuiría sobre todo a "mejorar la vida de la población". Probablemente, si se les hubiera hecho la pregunta directamente, más grupos se hubieran decantado por calificar a su actividad científica como una ciencia aplicada, pero al hacerles la pregunta de manera indirecta el propósito de la pregunta no fue tan evidente y reflejaron espontáneamente sus opiniones, quizá las más interiorizadas por su grupo de trabajo.

Todo ello nos lleva a reflexionar sobre una de las cuestiones más recurrentes de la ciencia, $i$ son los problemas de estudio los que deciden qué métodos hay que emplear o son los métodos los que modelan la manera en que se han de abordar los temas de estudio? $\mathrm{O}$, también, ¿son los objetos de estudio los que deciden qué metodologías y técnicas de investigación deben emplearse o, por el contrario, son las distintas metodologías las que aportan una manera concreta de enfocar ese objeto de estudio? La visión de los problemas cambia de una especialidad a otra, pero no parece que la elección de una técnica u otra defina su objeto de 
RIS

REVISTA INTERNACIONAL DE SOCIOLOGIA

№ 42, SEPTIEMBRE-DICIEMBRE, 2005

ANA M. GONZÁLEZ RAMOS Y TERESA GONZÁLEZ DE LA FE

estudio, puesto que son comunes a las distintas disciplinas y se emplean tanto por unas como por otras ciencias, aunque quizá con matizaciones significativas en su práctica cotidiana, las que tratamos de reflejar en este estudio caracterizándolas como sus principios de identidad. Por tanto, la conclusión es que no hay metodologías exclusivas de unas especialidades concretas, sino pautas de comportamiento que definen la manera en que se utiliza una herramienta de manera válida en una especialidad del conocimiento dada. Más aún, los grupos utilizan un gran número de metodologías, con finalidades muy variadas entre sí dependiendo de la naturaleza de su objeto de estudio o, incluso, para llevar a cabo partes diferentes de su estudio y cubrir objetivos distintos dentro de la misma investigación. Puede que la costumbre sea la que en mayor medida decida qué técnica se va a emplear y, sólo a partir de la experiencia de los investigadores, a la que se ha llegado mediante un largo proceso de socialización, se elijan finalmente los métodos más adecuados para emprender una investigación. Esta experiencia legitima la validez de la prueba y facilita la decisión de reconocimiento de la idoneidad de la prueba. En efecto, no es extraño observar en la práctica cotidiana que un equipo de investigación decide qué tipo de metodología va a emplear casi inmediatamente (si no previamente) a la elección del objeto de estudio.

Hemos visto que el área de problemas que un grupo de especialistas está dispuesto a abordar está sujeto a una constante modificación como resultado de la transformación de las corrientes científicas. "La complejidad es inevitable e inexorable: las células individuales se subdividen y se recombinan, buscan defender su integridad, en tanto cambian su forma y disposición" (Becher, 1989: 68), tal como es propio de un organismo vivo que necesita superarse constantemente y con esa superación sobrevivir a los cambios de su entorno y a la necesidad de ofrecer siempre nuevas respuestas. Rucio (cfr. Becher, 1989: 68) califica las especialidades científicas como una "mano de hierro" que controla el progreso de la ciencia y la profesión científica. También Toulmin (1977) se ha referido al asunto afirmando que cualquier tipo particular de cuestiones puede entrar dentro del dominio de varias ciencias diferentes, dependiendo del tipo de preguntas que se formulen acerca de él. La manera de abordar los objetos de estudio propios de cada disciplina tiene que ver con la forma en que ha sido construída socialmente por el conjunto de expertos legitimados de esa ciencia, que ha elegido los procedimientos básicos de la investigación y la forma "adecuada" de ver la naturaleza y las cuestiones fundamentales de esa especialidad científica. Un ejemplo de ello es la evolución sufrida por los estudios bioantropológicos que, según Haraway (1991: 154), sufrieron una importante trasformación a medida que el perfil de los investigadores aportaron una concepción distinta a su objeto de estudio y otra manera de poner en práctica su técnica de investigación básica: la observación de campo. Bachelard (2000:74) hace referencia a esta misma idea cuando introduce el concepto de técnica como elemento indispensable para separar la ciencia moderna de la ciencia precientífica. En su argumentación afirma que una ciencia no es tal 
hasta que es acompañada de una técnica específica de realización; la adopción de esta tecnología delimitadora no será posible sin la tradición, las normas, el consenso y las opiniones cualificadas de un grupo de científicos reconocidos, que aportan una seña de identidad al trabajo de sus colegas y una socialización específica dentro de ese campo de conocimiento (Campbell, 2003).

En cuanto a los factores que atañen a la orientación instrumental del trabajo científico es importante destacar que los procesos de cambio que afectan en la actualidad a la estructura de la ciencia han llevado a la trasformación de la idea general que se tiene de la labor científica. La ciencia se reorganiza con fórmulas nuevas, poniendo en cuestión las fronteras tradicionales entre las disciplinas (especialmente la división entre académicas y profesionales o públicas y privadas) y extendiendo prácticas que hasta ahora estaban restringidas a ciertos campos del conocimiento. Algunas normas y formas de trabajo, como los índices de impacto y la publicación en revistas extranjeras, se amplían al resto de las áreas e instituciones donde se produce el conocimiento científico. La multidisciplinariedad, la ciencia aplicada y la creación de vínculos de colaboración con la empresa privada se convierten en insignias de los nuevos tiempos (Bellavista et al., 1998). De todo ello hablaremos a continuación como complemento a los argumentos ofrecidos anteriormente para destacar el importante papel que desempeñan las culturas científicas en la actividad científica.

A lo largo de la historia de la ciencia se han establecido diversos criterios de clasificación para delimitar las etapas de transformación de la ciencia. Merton se refirió a la evolución de las ciencias desde el siglo XVII y durante todo el siglo XX dividiéndolas en tres momentos: la primera fase, amateur o periodo precientífico; la segunda, académica y, la tercera y última, post-académica. Esta tercera fase, con algunos cambios significativos, correspondería con lo que Gibbons y sus colaboradores (1997) denominaron Modo 2 o de producción socialmente distribuido, como contraposición a la actividad investigadora, tradicionalmente universitaria y de corte academicista. Existen otras clasificaciones, pero abundar en ellas no aportaría argumentos más sólidos a nuestro planteamiento, que se refiere al modo en que los cambios sociales influyen en la manera de organización de la ciencia y el trabajo desarrollado por la comunidad científica. Esos cambios son múltiples y bastante complejos, incluso diferentes según observemos unas u otras instituciones científicas, colectivos sociales y especialidades del conocimiento. En los últimos tiempos, se han desarrollado diversos estudios que abordan el estudio de los sistemas de I+D regionales, con la intención de comparar los efectos reales y el grado de difusión de esos cambios sobre la estructura científica y tecnológica, especialmente considerando las políticas de gestión y las normativas desarrolladas en cada región y sistema del conocimiento (para el caso de España puede consultarse Bellavista et al, 1998; González de la Fe, 2001; Romero et al., 2003; González Ramos, 2004; Olazaran et al., 2004). 
Olazaran et al. (2004) demuestran que el paso de un modelo a otro de ciencia (el que ellos denominan "segunda transición", similar a la descrita en el Modo 2) tiene unos efectos limitados entre los grupos de investigación del País Vasco. Los índices de producción y actividades de $\mathrm{I}+\mathrm{D}$ de esa región respecto al conjunto del territorio nacional es uno de los más favorables a pesar de que el tejido industrial de la región no se encuentra en sus momentos más álgidos; sin embargo, es superior al de la mayoría de las Comunidades Autónomas españolas. Por tanto, cabe esperar, que esos resultados tengan un efecto mayor que en el resto de las Comunidades Autónomas, es decir, si allí la transformación del nuevo sistema de conocimiento no está plenamente desarrollado, es lógico pensar que en la mayoría del territorio nacional tampoco lo estará, especialmente si la región es mucho más débil desde el punto de vista de su crecimiento económico y de la innovación. Cuanto más frágil sea un territorio, es decir, con un tejido industrial y empresarial menos sólido, más difícil será encontrar rasgos que lo sitúen dentro de la nueva economía del conocimiento. González Ramos (2004) ha encontrado en Cádiz una región en permanente situación de crisis industrial y desempleo estructural, resultados menos alentadores que los encontrados por Olazaran et al., (2004); es decir, una orientación menor hacia la realización de investigaciones aplicadas y escasas colaboraciones con las esferas industrial y empresarial del entorno. Este es allí un fenómeno incipiente, aunque no debe menospreciarse porque ha experimentado un crecimiento significativo en las últimas décadas, favorecida por los profundos cambios que afectan al panorama universitario español. Efectivamente, los vínculos con el sector privado han crecido como respuesta a un acontecimiento generalizado en todos los sistemas de ciencia y tecnología de precariedad. Esa situación les ha obligado a encontrar nuevas fuentes de financiación, pues los grupos ya no pueden abastecerse suficientemente del sector público, que ha reducido considerablemente las cantidades dedicadas a I+D. En consecuencia, se ha producido un incremento paulatino de las colaboraciones entre la universidad y la esfera privada, incluso a pesar de que la gran proporción de PYMEs no favorece, en absoluto, esta conectividad, puesto que el tamaño de la empresa ofrece más dificultades para asumir el riesgo de actividades innovadoras (Koschatzky y Sternberg, 2000).

Se achaca a los diferentes lenguajes de los ámbitos empresarial y universitario el escaso nivel de relaciones que se establece entre ellos, pues mientras la primera adopta una cultura de la inmediatez para afrontar sus resultados, la segunda tiene que contar con un desarrollo lento que tiene efectos sólo a medio y largo plazo (Fernández Esquinas, 2002: 54; Olazaran et al, 2004: 164-165). Sin embargo, la situación de crisis de la financiación pública favorece el cambio de percepción de los investigadores hacia la empresa y aumentan la probabilidad de colaboraciones puntuales o más duraderas, modificando sus líneas de trabajo hacia líneas más aplicadas o de interés para la empresa. La escasez presupuestaria ha hecho que los grupos de investigación hayan diversificado sus canales de financiación 
buscando fuentes alternativas, con la finalidad de obtener los recursos necesarios para desempeñar su trabajo (Bellavista et al., 1998: 101; Fernández Esquinas, 2002; González Ramos, 2004: 226-227; Olazaran et al., 2004). La nueva situación ha obligado a los equipos a aumentar el número de fuentes y a diversificar el abanico de entidades de las cuales puede proveerse de infraestructuras u otras necesidades presupuestarias de su equipo de investigación. Entre esas fuentes de financión alternativas, la empresa privada ha ocupado un lugar privilegiado que contribuye al desarrollo de la innovación y la investigación, ya sea como entidad que aporta la totalidad de la subvención o, más frecuentemente, como entidad cofinanciadora junto a algún organismo público. La necesidad de establecer una colaboración más estrecha entre ambas esfera no es el resultado, únicamente, del cambio de estrategia de los grupos de investigación, sino que está potenciada por las políticas públicas de gestión de la investigación y, consecuentemente, por el desarrollo de su normativa (Fernández Esquinas, 2002; Jiménez-Contreras, et al., 2003). El mensaje institucional, tanto europeo, como nacional y regional, insiste en que la actividad científica debe incrementar sus nexos de unión con el tejido empresarial y los datos avalan esa decisión, pues en todos los países se está produciendo, en mayor o menos medida.

Las nuevas reglas del juego determinan las decisiones tomadas por los investigadores acerca de los temas de estudio que han de elegir en el futuro, o la perspectiva de trabajo que adoptarán sobre la organización del trabajo del grupo de investigación (Becher, 1989: 180). Los cambios son sutiles, pero definitivos e influyen en la concepción que los actores principales tienen de su propio papel y de la actividad que desempeñan en este espacio social. Por ello, comienzan a emplear nuevas estrategias a la hora de plantearse sus líneas de investigación o los planes de crecimiento de sus empresas hacia esferas más innovadoras. Algunos grupos de investigación desarrollan dos líneas de investigación paralelas: unas "seguras", y otras "de riesgo" o, también, varias que combinan alternativamente para asegurarse varias vías de continuidad del estudio (Olazaran et al., 2004: 162). Los cambios son lentos, pero lo bastante notables como para ser perceptibles (por ejemplo, cuando su interés por los problemas que interesan a las empresas son abordados desde grupos de investigación universitarios que antes no se habrían acercado a esos temas). Las transformaciones que se están produciendo también afectan al mercado de trabajo científico (Fernández Esquinas, 2002), sobre todo añadiendo incertidumbres a la carrera profesional de los más jóvenes y modificando las relaciones de trabajo dentro de la comunidad científica. Bellavista et al. (1998) han destacado que el nuevo contexto ha afectado a las funciones tradicionales de los miembros de los grupos de investigación, incrementando el número de tareas administrativas y añadiendo otras adicionales, como la promoción de la actividad del grupo o la búsqueda de fuentes de financiación alternativas, de las que antes estaban libres. Estas nuevas funciones provocan una confrontación entre tareas, con los roles tradicionales y con el uso del tiempo que dedican a su ejercicio 
RIS

REVISTA INTERNACIONAL DE SOCIOLOCIA

profesional. Olazaran et al., (2004) han destacado otras cuestiones relativas al modo de organización del trabajo de los equipos de investigación universitarios. La dinámica de socialización (o formación del profesional investigador) está cambiando desde el modelo anterior, "modelo von Humboldt" en donde se combinaban las funciones de docencia y de investigación, hacia uno nuevo o "modelo Stanford" que se desarrollaría dentro de un contexto empresarial, sobre la base de la realización de investigaciones aplicadas (2004: 157). A la vista de estos datos y de los cambios normativos en la esfera universitaria, podemos señalar que el modelo de formación del personal investigador tradicional está llegando a su fin, tal y como lo perciben los mismos investigadores, quienes expresan sus dudas respecto al modo en que puede seguir sosteniéndose el sistema si no se protege a quienes tendrán que reemplazarlos algún día necesariamente.

La legislación actual y las condiciones de empleo en el mundo académico están acabando con el sistema tradicional de captación y reclutamiento de personal investigador (Fernández Esquinas, 2002). Es posible que dé lugar a uno nuevo, más o menos cercano al modelo Stanford, según cada contexto particular, o que ocurra de otra manera bien distinta a como lo estamos imaginando. Pero lo cierto es que la situación de incertidumbre y precariedad de la carrera profesional del científico desanima cada vez más a los más jóvenes a proseguirla, y los más veteranos ven en peligro su sistema de referencia, incluso respecto al modo habitual de desempeño de su trabajo cotidiano. A todo esto se añade la necesidad de buscar vías de financiación alternativas, que hace más complejas las tareas habituales (docentes, administrativas y de investigación) desempeñadas por los investigadores (Olazaran et al., 2004: 158). Pese a que todos estos cambios han redefinido los roles de los propios investigadores, la resistencia al cambio es bastante importante, puesto que algunos de esos nuevos valores entran en contradicción con los roles tradicionales. Así por ejemplo, al académico solitario puede parecerle totalmente improcedente hacer promoción de su trabajo o convertir sus líneas de investigación principales en algo útil o atractivo para la empresa porque considera su trabajo valioso por sí mismo. Esta reacción es una muestra de la resistencia que un grupo social puede protagonizar como defensa de su forma habitual de trabajo y como mantenimiento del status quo de su contexto social.

A todo ello viene a sumarse la percepción que los investigadores tienen de la valoración social de su trabajo por parte de la opinión pública en general. La manera de entender la profesión actualmente les hace ser más conscientes de la necesidad de ser evaluados positivamente por la sociedad y la necesidad de hacer lo posible para conectar estos dos ámbitos, la universidad y la sociedad, tan alejados hasta ahora. Los investigadores consideran que su trabajo es infravalorado y, asimismo, que ellos deberían incrementar sus esfuerzos de difusión de su trabajo. Pero eso supondría sumar otra tarea más a las de por sí ya numerosas y diversificadas tareas que han de realizar durante la misma jornada de trabajo. La universidad, aunque también puede extenderse a cualquier institución científica, 
se encuentra con dos obstáculos difíciles de superar en relación con este aspecto: por una parte, le corresponde estrechar los lazos con las empresas, de quienes depende su futuro financiero $\mathrm{y}$, por otra parte, debe fomentar el estrechamiento de los lazos con la sociedad a quien hasta ahora ha dejado caer en el olvido. En su segundo gran reto debe lograr superar la distancia que media entre ambas esferas sociales, favoreciendo las medidas de acercamiento y de promoción de la actividad científica, consiguiendo que su trabajo adquiera mayor relevancia para la opinión pública. Ello se traduce en un doble empeño: que sus resultados sean útiles para la sociedad y, a su vez, ésta los perciban de esa manera y, relacionado con lo anterior, que sus esfuerzos sean reconocidos por su propia valía, es decir, aunque de ellos no se desprendan ventajas inmediatas para la población. Todos esos elementos están relacionados con la comunicación y la difusión, dos tareas pendientes en la esfera de la ciencia.

\section{CONCLUSIONES}

Los argumentos expuestos demuestran una convergencia en cuanto al uso de una serie de metodologías en las distintas subdisciplinas científicas independientemente de su pertenencia a las ciencias naturales o a las sociales, poniendo en cuestión la división clásica entre las ciencias. También, las transformaciones que se están produciendo en el mundo de la ciencia afectan al modo en que se orienta el trabajo científico de los investigadores, lo que influye directamente en los resultados externos y en la organización interna de la actividad de los equipos de investigación. En cierto sentido, los métodos de trabajo tradicionales de las ciencias físicas y naturales se imponen con mayor fuerza sobre el resto de las áreas científicas, pero también se van imponiendo otros nuevos, que afectan a todas las áreas de investigación, como el necesario acercamiento hacia la esfera privada y empresarial, en busca de fuentes alternativas de financiación.

Hemos señalado la existencia de un fenómeno de mediatización de la actividad investigadora por parte de la cultura asumida por el investigador a través del proceso de socialización. Esta enculturación sería particular para cada subespecialidad y estaría sujeta a los vaivenes de la ciencia, creando un proceso de identificación de los miembros de la disciplina con las prácticas y valores relevantes para su campo de trabajo (Campbell, 2003). Esta cultura particular se plasma en los procedimientos y en las formas de actuación típicos de cada disciplina, convirtiéndose en estilos diferentes de trabajo de cada especialidad científica, avalada con técnicas de investigación que son comunes a varias disciplinas, lo que invalida los discursos defensores de la rígida división entre ciencias blandas y ciencias duras (o fisico-naturales y sociales) respecto a la utilización de un método de trabajo exclusivo. Los límites entre la forma en que se emplean los métodos de investigación y los medios para difundir los resultados científicos son sutiles y 
variables incluso dentro de la misma especialidad del conocimiento. Además, una referencia a la historia de las disciplinas muestra que sus límites son difusos y cambiantes a pesar de que aporten rasgos peculiares y distintivos de la identidad de esa disciplina científica.

Por otra parte, el estilo de trabajo derivado de su cultura particular anularía el efecto de otras variables demográficas como el género que, sin embargo, ocupan más la atención de los sociólogos de la ciencia. En cambio, la variable edad ofrece una lectura bastante distinta, pues refleja la transformación de las comunidades científicas debido a la presión ejercida sobre su contexto profesional y su mayor disposición a absorber los cambios de la ciencia mucho más fácilmente. Los jóvenes se muestran más activos y próximos a los nuevos modos de proceder en su campo de conocimiento, conforme los cambios se van introduciendo en las estructuras de los propios grupos de investigación. Ejemplo de ello sería la orientación más decidida hacia el mercado y la investigación aplicada o la multiplicación de las tareas asociadas a la labor científica.

$\mathrm{La}$ aportación original de este trabajo no es haber descubierto la influencia de la cultura en las comunidades científicas, sino haber profundizado en su análisis, abordando aspectos que hasta ahora habían sido descuidados, como, por ejemplo, el empleo de técnicas de investigación por parte de las especialidades científicas. La exposición de estos datos nos ha llevado a reflexionar sobre los efectos causados por los cambios normativos y de la gestión política de la esfera científica. Estos factores contribuyen a la transformación del modo de trabajo de las comunidades científicas, ya que al imponerse nuevos procedimientos de evaluación científica y de planificación de la I+D se eligen otros métodos distintos para difundir los resultados de la investigación (el tipo de documentos o los de espacios donde se publican). Del mismo modo, los cambios contextuales implican una manera novedosa de entender la investigación, desde un punto de vista práctico y desde un punto de vista global, que afecta tanto a la ciencia como a las entidades privadas y a la sociedad en general.

\section{REFERENCIAS BIBLIOGRÁFICAS}

BACHELARD, G. (2000), La formación del espiritu cientifico. Contribución a un psicoanálisis del conocimiento objetivo, Madrid, Siglo XXI.

BARRAL, M. J. et al, (eds.) (1999), Interacciones ciencia y género. Discursos y prácticas cientificas de mujeres, Barcelona, Icaria.

BECHER, T. (1989), Tribus y territorios académicos. La indagación intelectual y las culturas de las disciplinas, Barcelona, Gedisa.

BELLAVISTA, J., T. TURPIN, S. HILL y de J.M. MIGUEL, (1998), "Cultura organizativa de investigadores y entorno político y social”, Papers, $\mathrm{n}^{\circ}$ 54, pp. 79-109. 
BIJKER, W. E. (1997), "La construcción social de la baquelita: Hacia una teoría de la invención" en M.I. González, J.A. López, J.L. Luján, (eds.), Ciencia, Tecnologia y Sociedad, Barcelona, Ariel.

BOURDIEU, P. (2003), El oficio de cientifico. Ciencia de la ciencia y reflexividad, Barcelona, Anagrama.

BOZEMAN, B. y J. MANSEMATIN (2004), "Editor's Introduction: Building and deploying scientific and technical human capital", Research Policy, n 33, pp. 565-568.

CAMPBELL, R.A. (2003), "Preparing the next generation of scientists: The social process of managing students", Social Studies of Science, $\mathrm{n}^{\circ} 33,6,897-927$.

CRANE, D. (1972), Invisible Colleges, Chicago, University Press.

CHAVOYA, M.L. (2001), “Organización del trabajo y culturas académicas. Estudio de dos grupos de investigadores de la Universidad de Guadalajara", Revista Mexicana de Investigación Educativa, vol. 6, (11), pp. 79-93.

DASGUPTA, P. y P.A. DAVID (1985), Information disclosure and economic of science and technology, Publication, $\mathrm{n}^{\circ} \mathrm{48}$, Stanford, Stanford University Centre for Economic Policy Research.

FERNÁNDEZ ESQUINAS, M. (2002), "Mercados de trabajo en la ciencia. Balance de la investigación y propuesta de marco analítico", Revista Internacional de Sociologia, n⿳ 32, pp.35-75.

(2002), La formación de investigadores cientificos en España, Madrid, Centro de Investigaciones Sociológicas.

GIBBONS, M. et al, (1997), La nueva producción del conocimiento. La dinámica de la ciencia y la investigación en las sociedades contemporáneas, Barcelona, Pomares-Corredor.

GONZÁLEZ DE LA FE, T., M. PÉREZ YRUELA y M. FERNÁNDEZ ESQUINAS (1996), "La formación de investigadores en el Plan Nacional de I+D: Una aproximación evaluativa", Gestión y Análisis de Politicas Públicas, $\mathrm{n}^{\circ}$. 5-6, pp. 125-142.

GONZÁLEZ DE LAFE, T. (2001), "Las políticas regionales de ciencia, tecnología e innovación. Análisis comparativo", $V$ Congreso Español de Ciencia Política y de la Administración, septiembre.

GONZÁLEZ RAMOS, A.M. (2004), Modos de producción cientifica: culturas y metodologias de investigación en la Universidad de Cádiz, Tesis doctoral, Universidad de Cádiz (en prensa).

HANKINS, T. L. (1988), Ciencia e Ilustración, Madrid, Alianza.

HARAWAY, D. J. (1991), Ciencia, cyborgs y mujeres. La reinvención de la naturaleza, Valencia, Cátedra.

KING, G., R.O. KEOHANE y S. VERBA (2000), El diseño de la investigación social. La inferencia cientifica en los estudios cualitativos, Madrid, Alianza. 
RIS

REVISTA INTERNACIONAL DE SOCIOLOCIA

KOSCHATZKY y STERNBERG (2000), "R\&D Cooperation innovation systems -Some lessons from European Innovation Survey (ERIS)", European Planning Studies, vol. 8, nº 4.

KOYRÉ, A. (1981), Estudios galileanos, México, Siglo XXI.

KUHN, T.S. (1977), La tensión esencial. Estudios selectos sobre la tradición y el cambio en el ámbito de la ciencia, México, Fondo de Cultura Económica.

JIMÉNEZ-CONTRERAS, E., F. MOYA, E. DELGADO (2003), "The evolution research activity in Spain. The impact of the National Comission for the Evaluation of Research Activity (CNEAI)", Research Policy, 32, pp. 123-142.

LONG, J.S. y M.F. FOX (1995) "Scientific Careers: Universalism and Particularism" Annual Review Sociology, Vol. 21, pp. 45-71.

LÓPEZ PIÑERO, J.M. (2000), Breve Historia de la Medicina, Madrid, Alianza.

MERTON, R. K. (1938), Science, technology and society in Seventeenth-Century England, Nueva York, Howard Ferting.

(1973), The Sociology of Science. Theoretical and empirical investigations, Chicago, University of Chicago Press.

(2002), "La ciencia y el orden social", en Teoría y estructura sociales, México, Fondo de Cultura Económica.

MULKAY, M. (1977), "Sociology of the scientific community”, en Spielgel-Rösing y Price (comps.) Science, technology and Society, Beverly Hills, Sage.

OLAZARAN, M., C. LAVÍA Y B. OTERO (2004), “Hacia una segunda transición en la ciencia? Política científica y grupos de investigación”, $R E S \mathrm{n}^{\circ} 4$, pp. 143-172.

ROMERO, M., L. CRUZ y L. SANZ MENÉNDEZ (2003), "Estabilidad en las políticas andaluzas de ciencia, tecnología e innovación", Revista Internacionacional de Sociología, $\mathrm{n}^{\circ} 35$, pp. 7-51.

RUSCIO, K.P. (1987), Many sectors, many professions, en B.R. Clark (comp.), The academic profession, Berkerley, University Academic Press.

SANZ, L., M. BORDONS y L.M. ZULUETA (2001) "Interdisciplinarity as a multidimensional concept: its measure in three different research areas", Unidad de Politicas Comparadas, CSIC, working paper 01-04.

SEDAITIS, J. (2000), "Technology transfer in transitional economies: a test of market, state and organizational models", Research Policy, 29, PP. 135-147.

SHAPITA, P., H. KLEIN y S. KUHLMANN (2001), "Innovations in European and US innovation policy", Research Policy, 30, pp. 869-872.

STORER, N. W. (1976), "The hard sciences and the soft: Some sociological observations", Bulletin of the Medical Library Association, 55 (1), pp. 75-84. 
TORRES, C. (1989), Sociologia politica de la ciencia, Madrid, Centro de Investigaciones Sociológicas.

TOULMIN, S. (1977) La comprensión humana, Madrid, Alianza.

WHITLEY, R.D. (1974), The social processes of Scientific Development, London, Routledge and Kegan.

WRAY, K.B. (2005), “Rethinking scientific specialization”, Social Studies of Science, 35/1, pp. 151-164. 